\title{
Decision Support Methodology Based on Covering-Based Interval-Valued Pythagorean Fuzzy Rough Set Model and Its Application to Hospital Open-Source EHRs System Selection
}

\author{
Fang Liu, ${ }^{1}$ Ju Wu, ${ }^{2}$ Lianming Mou, ${ }^{2}$ and Yi Liu $\mathbb{D}^{1,2,3}$ \\ ${ }^{1}$ Data Recovery Key Laboratory of Sichuan Province, Neijiang Normal University, Neijiang 641000, Sichuan, China \\ ${ }^{2}$ School of Mathematics and Information Sciences, Neijiang Normal University, Neijiang 641000, Sichuan, China \\ ${ }^{3}$ Numerical Simulation Key Laboratory of Sichuan Province, Neijiang Normal University, Neijiang 641000, Sichuan, China \\ Correspondence should be addressed to Yi Liu; liuyiyl@126.com
}

Received 18 March 2020; Revised 17 April 2020; Accepted 22 April 2020; Published 11 May 2020

Guest Editor: Zaoli Yang

Copyright (C) 2020 Fang Liu et al. This is an open access article distributed under the Creative Commons Attribution License, which permits unrestricted use, distribution, and reproduction in any medium, provided the original work is properly cited.

\begin{abstract}
The target of current work is to propose a new approach to deal with multiattribute decision-making (MADM) problems with interval-valued Pythagorean fuzzy set (IVPFS) based on the concepts of covering-based rough set (CRS) and TOPSIS and give its application in MADM problems. To begin with, we integrate the fuzzy rough set (FRS), IVPFS and CRS and define the coveringbased interval-valued Pythagorean fuzzy rough set (CIVPFRS). Firstly, the relative notions of the CIVPFRS model are introduced. In addition, the distance measure of interval-valued Pythagorean fuzzy numbers (IVPFNs) is defined; based on the proposed distance, the rough and precision degrees of CIVPFRS are discussed. Thirdly, on the basis of the theoretical analysis for CIVPFRS models, an interval-valued Pythagorean fuzzy TOPSIS method is designed to deal with the MADM problems with interval-valued Pythagorean fuzzy information (IVPFI). Last of all, the validity and merits of the proposed approach are illustrated by an example, and the sensitivity analysis of the parameters and the comparison with the existing related methods are carried out. $\beta$
\end{abstract}

\section{Introduction}

Fuzzy set theory (FS) [1] and rough set theory (RS) [2] are both used to address some problems with uncertainty. RS describes the target set by two definite sets, which are upper approximation set and lower approximation set. The description process does not need any prior knowledge, it is completely based on the given data for analysis and judgment, so RS has been employed in many fields, for instance, data mining, artificial intelligence, decision analysis, and many other fields. Different from RS, FS needs prior knowledge to describe the object, such as expert experience, which has better applicability. There is a strong complementarity between RS and FS. Therefore, the integration of the two has become a new research hotspot, which has aroused the research interest of scholars [1,3-6].

Intuitionistic fuzzy sets (IFSs) [7] are an effective extension of FSs, which can describe the fuzziness of the objective world from three aspects: support, opposition, and neutrality. As an IFS can consider both the membership degree (MD) and nonmembership degree (NMD) of elements belonging to the set at the same time, therefore, IFS is widely concerned by decision makers and fruitful results have been achieved. Wan and Dong [8] studied the theory and method of decision-making based on interval-valued intuitionistic fuzzy sets. However, IFSs have some limitations in the application of MADM. For example, it can only describe the fuzzy phenomenon that the sum of MD and NMD is not more than one, but it cannot do anything to the phenomenon that the sum of MD and NMD is more than one. For this reason, Yager and Abbasov [9] put forward the Pythagorean fuzzy set (PFS) to solve the abovementioned limitations. The main difference between IFS and PFS is that, in PFS the sum of squares of MD and NMD are real numbers between zero and one. Based on Yager's research, many scholars have studied the PFS and obtained some research 
results. Among them, Garg [10] developed some new operational laws and their corresponding weighted geometric aggregation operators. Garg [11] extended the traditional Maclaurin symmetric mean operator to hesitant Pythagorean fuzzy environment. Liu et al. [12] constructed the multiattribute group decision-making (MAGDM) approach with linguistic Pythagorean fuzzy (LPF) information based on generalized LPF aggregation operators. Then, Liu et al. [13] investigated MADM problems with Pythagorean linguistic information based on some new aggregation operators. Zhang and Xu [14] studied the TOPSIS method under PFS environment; Akram et al. [15] extended the TOPSIS method to solve MCGDM problems with Pythagorean fuzzy data. Wan et al. [16] developed a three-phase method for solving MAGDM using PFNs. Wan et al. [17] also developed a PF mathematical programming method to solve the MAGDM problem in the PF environment. Then, Wan et al. [18] proposed a new order relation for PFNs and applies to MAGDM. In order to express the more complicated uncertainty information, Peng and Yang [19] extended the PFS to the IVPFS and built decision approach for MADM problems with IVPFI based on some proposed aggregation operators. After that, some research studies on IVPFS have emerged in succession. Such as Wei et al. [20] established some MADM methods based on proposed interval-valued Pythagorean fuzzy (IVPF) Maclaurin symmetric average operator and IVPF-weighted Maclaurin symmetric average operator. Garg [21] presented the mean and geometric aggregation operators in interval-valued Pythagorean fuzzy environment. Khan and Abdullah [22] introduced the concept of IVPF Choquet integral average operator. Wang et al. [23] proposed interval-valued hesitant Pythagorean fuzzy sets (IVHPFSs) and investigated some properties of IVHPSs.

Zakowski's [24] CRS is a generalization model of the classical RS. It is an extension of the partition of Pawlak RS to the covering of RS. On this basis, two rough approximation operators are constructed and many conclusions are obtained. After that many scholars investigated many kinds of RS models based on covering from different angles. In 2003, Zhu and Wang [25] put forward the generalized rough set model of covering and studied the reduction and axiomatic properties of the model. Then, they put forward three different types of CRS models based on the known models and described many important properties. In 2016, Safari and Hooshmandasl [26] introduced twelve kinds of coverage approximation operators and studied the structural properties and interrelations of these twelve types of CRS models. Furthermore, Ma [27] replaces the classical equivalence relation with the general binary relation (neighborhood relation), thus generalizes the CRS. In recent years, many scholars have extended the classical CRS to the fuzzy environment. Dubios and Prade [28] presented the rough fuzzy set (RFS) and fuzzy rough set (FRS). Researchers have carried out some research studies on CFRS. Ma [29] introduced the generalized structure of CFRS. Deer et al. $[30,31]$ presented the concept of fuzzy $\beta$-neighborhoods and fuzzy neighborhoods. Hussain et al. [32] presented q-rung orthopair fuzzy TOPSIS (q-ROF-TOPSIS) methodology for the MADM problem which depends on the covering-based q-rung orthopair fuzzy sets (Cq-ROFRSs) model. Zeng et al. [33] proposed a method to solve MADM problem based on covering-based spherical fuzzy rough set (CSFRS) models and built TOPSIS method to deal with MADM problem. Hussain et al. [34] presented the notions of rough $\mathrm{Py}-$ thagorean fuzzy ideals in semigroups. Zheng et al. [4] put forward the extended uncertainty measurement criterion of CRIFSs and introduced an improved roughness method. By combining PFS and FRS, Zhang and Li [5] put forth the concept of Pythagorean fuzzy rough set (PFRS).

As can be seen from the latest hot research directions, the CIVPFS model is an important tool for dealing with uncertainty in the real world. Therefore, it is necessary to build the CIVPFRS model by integrating the IVPFS and CFRS in order to deal with some information with more complicated uncertainty. As far as we know, there is no concept of IVPF rough set in IVPF $\beta$-neighborhood systems. In order to fill this research space, this paper attempts to study CIVPFRS model through IVPF $\beta$-neighborhood systems. This paper proposes a new approach to deal with MADM problems with IVPFS information based on the concepts of CRS and TOPSIS and gives its application in MADM problem. In real life, CIVPFRS model is a vital tool to handle complexity and uncertainty. Based on the concept of CRS, IVPFS, and FRS, the idea of building CIVPFRS model by IVPF $\beta$-neighborhoods is studied. In addition, by adjusting the value of $0 \leq\left(\mu_{U}(x)\right)+\left(\nu_{U}(x)\right) \leq 1$, it is found that CIVPFRS is an important extension of cover-based interval-valued intuitionistic fuzzy rough set (CIVIFRS). By adjusting the value of $\mu_{L}=\mu_{U}$ and $\nu_{L}=\nu_{U}$, it is an important extension of coverbased Pythagorean fuzzy rough set (CPFRS). By adjusting $\mu_{L}=\mu_{U}, \nu_{L}=\nu_{U}$, and $0 \leq\left(\mu_{U}(x)\right)+\left(\nu_{U}(x)\right) \leq 1$, it is an important extension of cover-based intuitionistic fuzzy rough set (CIFRS). This shows that CIVPFRS model has stronger ability to deal with uncertainty than IFS, intervalvalued intuitionistic fuzzy set (IVIFS), and PFS.

Based on the abovementioned ideas, the present paper introduces the CIVPFRS model based on IVPF $\beta$-neighborhoods and its utilizations in MADM problem. The rest of this paper is arranged as follows: the basic concepts of PFS and IVPFS and their generalization are introduced in Section 2. In Section 3, the concept of CIVPFRS model based on IVPF $\beta$-neighborhoods is proposed along with the corresponding axiomatic system. Apart from these, the roughness and precision degrees of CIVPFRS model are also mainly discussed in this section. In Section 4, based on the analysis of CIVPFRS model, we introduce the interval-valued Pythagorean fuzzy TOPSIS (IVPF-TOPSIS) method to solve the MADM problem with IVPFI. Furthermore, we also construct a method based on CIVPFRS model to solve the MADM problem with IVPFI and design the corresponding algorithm. In Section 5, an example of practical application is provided to show how IVPF-TOPSIS can deal with MADM problems by using the concept of CIVPFRS model based on IVPF $\beta$-neighborhoods. Moreover, the sensitivity analysis of the parameters and the correctness analysis of the results are carried out and the conclusion will be obtained in Section 6. 


\section{Preliminaries}

Basic concepts and notations of PFS and IVPFS are outlined in this section.

Definition 1 (see [9]). Let $U$ be a finite universe set. The PFS $P$ of $U$ is defined as follows:

$$
P=\left\{\left\langle y, \mu_{P}(y), v_{P}(y)\right\rangle \mid y \in U\right\}
$$

where $0 \leq \mu_{P} \leq 1$ and $0 \leq \nu_{P} \leq 1$ denote the MD and NMD of $y \in U$ to the set $P$, which satisfy $0 \leq \mu_{P}^{2}(y)+v_{P}^{2}(y) \leq 1$ for all $y \in U . \pi_{P}(y)=\sqrt{1-\mu_{P}^{2}(y)-v_{P}^{2}(y)}$ represents the degree of indeterminacy of $y$ to $P$ and called the indeterminacy degree.

Definition 2 (see [19]). Let $U$ be a finite universe set. For every $y \in U, \mu_{P}(y)$ and $\nu_{P}(y)$ are closed intervals, that is, $\mu_{P}(y)=\left[\mu_{L}(y), \mu_{U}(y)\right]$ and $\nu_{P}(y)=\left[\nu_{L}(y), \nu_{U}(y)\right]$. Then, an IVPFS IVP in $U$ is defined as follows:

$$
\operatorname{IVP}=\left\{\left\langle y,\left(\left[\mu_{L}(y), \mu_{U}(y)\right],\left[\nu_{L}(y), v_{U}(y)\right]\right)\right\rangle \mid y \in U\right\},
$$

where $0 \leq\left(\mu_{U}(y)\right)^{2}+\left(\nu_{U}(y)\right)^{2} \leq 1$. Then, the degree of indeterminacy is defined as $\pi_{\mathrm{IVP}}(y)=\left[\pi_{L}(y), \pi_{U}(y)\right]=$ $\left[\sqrt{1-\mu_{U}^{2}(y)-v_{U}^{2}(y)}, \sqrt{1-\mu_{L}^{2}(y)-v_{L}^{2}(y)}\right] . \quad$ Denoted $\operatorname{IVP}=\operatorname{IVP}\left(\left[\mu_{L}, \mu_{U}\right],\left[\nu_{L}, \nu_{U}\right]\right)$ as an IVPFN, where $\left[\mu_{L}, \mu_{U}\right] \subseteq[0,1]$ and $\left[v_{L}, v_{U}\right] \subseteq[0,1]$. In the following, we use $\operatorname{IVPF}(U)$ to denote all IVPFSs on $U$.

The score function and accuracy function used to compare two IVPFNs are defined as follows:

Definition 3 (see [19]). For an $\operatorname{IVPFN} \operatorname{IVP}=\operatorname{IVP}\left(\left[\mu_{L}\right.\right.$, $\left.\left.\mu_{U}\right],\left[\nu_{L}, v_{U}\right]\right)$, the score function of IVP is defined as follows:

$$
s(\mathrm{IVP})=\frac{1}{2}\left(\mu_{L}^{2}+\mu_{U}^{2}-v_{L}^{2}-v_{U}^{2}\right)
$$

where $-1 \leq s($ IVP $) \leq 1$. The accuracy function of $\operatorname{IVP}=\operatorname{IVP}\left(\left[\mu_{L}, \mu_{U}\right],\left[\nu_{L}, \nu_{U}\right]\right)$ can be defined as follows:

$$
a(\operatorname{IVP})=\frac{1}{2}\left(\mu_{L}^{2}+\mu_{U}^{2}+\nu_{L}^{2}+\nu_{U}^{2}\right)
$$

where $0 \leq a(\operatorname{IVP}) \leq 1$.

For any IVPFN $P_{A}, P_{B}$ of $U$, we can compare two IVPFNs by the following rules:

(1) If $s\left(P_{A}\right)>s\left(P_{B}\right)$, then $P_{A}$ is bigger than $P_{B}$ and is denoted by $P_{A}>P_{B}$.

(2) If $s\left(P_{A}\right)=s\left(P_{B}\right)$, then

(i) If $a\left(P_{A}\right)>a\left(P_{B}\right)$, then $P_{A}>P_{B}$.

(ii) If $a\left(P_{A}\right)=a\left(P_{B}\right)$, then $P_{A} \sim P_{B}$.

\section{CIVPFRS Model}

In this section, we introduce the concept of CIVPFRS.

Definition 4. (1) Assume $U$ is an universe set, $\widetilde{E}=\left\{\widetilde{E}_{1}, \widetilde{E}_{2}, \ldots, \widetilde{E}_{n}\right\}$, where $\widetilde{E} \in \operatorname{IVPF}(U)$ and $k=1, \ldots, n$. For any IVPFN $\beta=\left\langle\left[s_{L}, s_{U}\right],\left[t_{L}, t_{U}\right]\right\rangle$, then $\widetilde{E}$ is called an interval-valued Pythagorean fuzzy $\beta$-covering (IVPF $\beta$-covering) of $U$ if

$$
\left(\bigcup_{k=1}^{n} \widetilde{E}_{k}\right)(y) \geq \beta,
$$

for all $y \in U$. The $(U, \widetilde{E})$ is called an interval-valued Pythagorean fuzzy covering approximation space (IVPFCAS).

(2) Let $(U, \widetilde{E})$ be an IVPFCAS and $\widetilde{E}=\left\{\widetilde{E}_{1}, \widetilde{E}_{2}, \ldots, \widetilde{E}_{n}\right\}$ be an IVPF $\beta$ - covering of $U$ for some $\beta=\left\langle\left[s_{L}\right.\right.$, $\left.\left.s_{U}\right],\left[t_{L}, t_{U}\right]\right\rangle$. Then,

$$
\widetilde{N}_{\widetilde{E}(y)}^{\beta}=\bigcap\left\{\widetilde{E}_{k} \in \widetilde{E} \mid \widetilde{E}_{k}(y) \geq \beta, k=1, \ldots, n\right\}
$$

is called an IVPF $\beta$-neighborhood of $y$ in $U$.

Let $\widetilde{N}_{\widetilde{E}}^{\beta}=\left\{\widetilde{N}_{\widetilde{E}(y)}^{\beta} \mid y \in U\right\}$ represent IVPF $\beta$-neighborhood system induced by IVPF $\beta$-covering $\widetilde{E}$. The IVPF $\beta$-neighborhood system is represented by the IVPF matrix as follows:

$$
\tilde{M}_{\widetilde{E}}^{\beta}=\left[\widetilde{N}_{\widetilde{E}(y)}^{\beta}(z)\right]_{(y, z) \in U \times U} .
$$

Example 1. Suppose that $(U, \widetilde{E})$ is an IVPFCAS and $\widetilde{E}=$ $\left\{\widetilde{E}_{1}, \widetilde{E}_{2}, \ldots, \widetilde{E}_{5}\right\}$ is a set of IVPFS, where $U=\left\{y_{1}, y_{2}, \ldots, y_{6}\right\}$, $\beta=\langle[0.3,0.5],[0.3,0.4]\rangle$. Details are shown in Table 1 .

Therefore, $\widetilde{E}$ is an IVPF $\beta$-covering of $U$. Then,

$$
\begin{aligned}
& \widetilde{N}_{\widetilde{E}\left(y_{1}\right),}^{\langle[0.3,0.5],[0.3,0.4]\rangle}=\widetilde{E}_{1} \cap \widetilde{E}_{4}, \\
& \widetilde{N}_{\widetilde{E}\left(y_{2}\right)}^{\langle[0.3,0.5],[0.3,0.4]\rangle}=\widetilde{E}_{1} \cap \widetilde{E}_{3}, \\
& \widetilde{N}_{\tilde{E}\left(y_{3}\right)}^{\langle[0.3,0.5],[0.3,0.4]\rangle}=\widetilde{E}_{1} \cap \widetilde{E}_{5}, \\
& \widetilde{N}_{\widetilde{E}\left(y_{4}\right)}^{\langle[0.3,0.5],[0.3,0.4]\rangle}=\widetilde{E}_{1} \cap \widetilde{E}_{5}, \\
& \widetilde{N}_{\widetilde{E}\left(y_{5}\right)}^{\langle[0.3,0.5],[0.3,0.4]\rangle}=\widetilde{E}_{2} \cap \widetilde{E}_{3}, \\
& \widetilde{N}_{\widetilde{E}\left(y_{6}\right)}^{\langle[0.3,0.5],[0.3,0.4]\rangle}=\widetilde{E}_{2} \cap \widetilde{E}_{4} .
\end{aligned}
$$

By calculations, we have the $\tilde{N}_{\tilde{E}}^{\langle[0.3,0.5],[0.3,0.4]\rangle}$, as shown in Table 2. Then, we get the IVPF matrix and listed as follows. That is, 
TABle 1: IVPF $\beta$-covering $\widetilde{E}$ in Example 1.

\begin{tabular}{lccccc}
\hline$U / \widetilde{E}$ & $\widetilde{E}_{1}$ & $\widetilde{E}_{2}$ & $\widetilde{E}_{3}$ & $\widetilde{E}_{4}$ & $\widetilde{E}_{5}$ \\
\hline$y_{1}$ & $\langle[0.6,0.8],[0.1,0.2]\rangle$ & $\langle[0.5,0.7],[0.5,0.6]\rangle$ & $\langle[0.3,0.5],[0.7,0.8]\rangle$ & $\langle[0.6,0.9],[0.1,0.3]\rangle$ & $\langle[0.3,0.4],[0.3,0.6]\rangle$ \\
$y_{2}$ & $\langle[0.4,0.7],[0.2,0.3]\rangle$ & $\langle[0.2,0.4],[0.1,0.5]\rangle$ & $\langle[0.4,0.8],[0.1,0.4]\rangle$ & $\langle[0.5,0.6],[0.4,0.7]\rangle$ & $\langle[0.2,0.7],[0.1,0.2]\rangle$ \\
$y_{3}$ & $\langle[0.3,0.6],[0.3,0.4]\rangle$ & $\langle[0.7,0.8],[0.4,0.6]\rangle$ & $\langle[0.2,0.8],[0.2,0.3]\rangle$ & $\langle[0.1,0.3],[0.6,0.9]\rangle$ & $\langle[0.6,0.7],[0.1,0.2]\rangle$ \\
$y_{4}$ & $\langle[0.5,0.8],[0.1,0.3]\rangle$ & $\langle[0.2,0.3],[0.6,0.9]\rangle$ & $\langle[0.1,0.4],[0.1,0.3]\rangle$ & $\langle[0.4,0.7],[0.3,0.6]\rangle$ & $\langle[0.4,0.8],[0.1,0.4]\rangle$ \\
$y_{5}$ & $\langle[0.1,0.2],[0.5,0.7]\rangle$ & $\langle[0.3,0.7],[0.1,0.3]\rangle$ & $\langle[0.8,0.9],[0.1,0.2]\rangle$ & $\langle[0.6,0.7],[0.2,0.5]\rangle$ & $\langle[0.1,0.8],[0.2,0.3]\rangle$ \\
$y_{6}$ & $\langle[0.4,0.6],[0.4,0.6]\rangle$ & $\langle[0.3,0.7],[0.1,0.2]\rangle$ & $\langle[0.5,0.6],[0.3,0.5]\rangle$ & $\langle[0.7,0.8],[0.1,0.3]\rangle$ & $\langle[0.1,0.4],[0.1,0.2]\rangle$ \\
\hline
\end{tabular}

TABLe 2: $\widetilde{N}_{\tilde{E}}^{\langle[0.3,0.5],[0.3,0.4]\rangle}$ in Example 1.

\begin{tabular}{ccccccc}
\hline$\widetilde{N}_{\tilde{E}}^{\beta}$ & $y_{1}$ & $y_{2}$ & $y_{3}$ & $y_{4}$ & $y_{5}$ & $y_{6}$ \\
\hline$y_{1}$ & $\langle[0.6,0.8],[0.1,0.3]\rangle$ & $\langle[0.4,0.6],[0.4,0.7]\rangle$ & $\langle[0.1,0.3],[0.6,0.9]\rangle$ & $\langle[0.4,0.7],[0.3,0.6]\rangle$ & $\langle[0.1,0.2],[0.5,0.7]\rangle$ & $\langle[0.4,0.6],[0.4,0.6]\rangle$ \\
$y_{2}$ & $\langle[0.3,0.5],[0.7,0.8]\rangle$ & $\langle[0.4,0.7],[0.2,0.4]\rangle$ & $\langle[0.2,0.6],[0.3,0.4]\rangle$ & $\langle[0.1,0.4],[0.1,0.3]\rangle$ & $\langle[0.1,0.2],[0.5,0.7]\rangle$ & $\langle[0.4,0.6],[0.4,0.6]\rangle$ \\
$y_{3}$ & $\langle[0.3,0.4],[0.3,0.6]\rangle$ & $\langle[0.2,0.7],[0.2,0.3]\rangle$ & $\langle[0.3,0.6],[0.3,0.4]\rangle$ & $\langle[0.4,0.8],[0.1,0.4]\rangle$ & $\langle[0.1,0.2],[0.5,0.7]\rangle$ & $\langle[0.1,0.4],[0.4,0.6]\rangle$ \\
$y_{4}$ & $\langle[0.3,0.4],[0.3,0.6]\rangle$ & $\langle[0.2,0.7],[0.2,0.3]\rangle$ & $\langle[0.3,0.6],[0.3,0.4]\rangle$ & $\langle[0.4,0.8],[0.1,0.4]\rangle$ & $\langle[0.1,0.2],[0.5,0.7]\rangle$ & $\langle[0.1,0.4],[0.4,0.6]\rangle$ \\
$y_{5}$ & $\langle[0.3,0.5],[0.7,0.8]\rangle$ & $\langle[0.2,0.4],[0.1,0.5]\rangle$ & $\langle[0.2,0.8],[0.4,0.6]\rangle$ & $\langle[0.1,0.3],[0.6,0.9]\rangle$ & $\langle[0.3,0.7],[0.1,0.3]\rangle$ & $\langle[0.3,0.6],[0.3,0.5]\rangle$ \\
$y_{6}$ & $\langle[0.5,0.7],[0.5,0.6]\rangle$ & $\langle[0.2,0.4],[0.4,0.7]\rangle$ & $\langle[0.1,0.3],[0.6,0.9]\rangle$ & $\langle[0.2,0.3],[0.6,0.9]\rangle$ & $\langle[0.3,0.7],[0.2,0.5]\rangle$ & $\langle[0.3,0.7],[0.1,0.3]\rangle$ \\
\hline
\end{tabular}

$M_{\widetilde{E}}^{\langle[0.3,0.5],[0.3,0.4]\rangle}=\left(\begin{array}{llll}\langle[0.6,0.8],[0.1,0.3]\rangle & \langle[0.4,0.6],[0.4,0.7]\rangle\langle[0.1,0.3],[0.6,0.9]\rangle\langle[0.4,0.7],[0.3,0.6]\rangle\langle[0.1,0.2],[0.5,0.7]\rangle\langle[0.4,0.6],[0.4,0.6]\rangle \\ \langle[0.3,0.5],[0.7,0.8]\rangle\langle[0.4,0.7],[0.2,0.4]\rangle\langle[0.2,0.6],[0.3,0.4]\rangle\langle[0.1,0.4],[0.1,0.3]\rangle\langle[0.1,0.2],[0.5,0.7]\rangle\langle[0.4,0.6],[0.4,0.6]\rangle \\ \langle[0.3,0.4],[0.3,0.6]\rangle\langle[0.2,0.7],[0.2,0.3]\rangle\langle[0.3,0.6],[0.3,0.4]\rangle\langle[0.4,0.8],[0.1,0.4]\rangle\langle[0.1,0.2],[0.5,0.7]\rangle\langle[0.1,0.4],[0.4,0.6]\rangle \\ \langle[0.3,0.4],[0.3,0.6]\rangle\langle[0.2,0.7],[0.2,0.3]\rangle\langle[0.3,0.6],[0.3,0.4]\rangle\langle[0.4,0.8],[0.1,0.4]\rangle\langle[0.1,0.2],[0.5,0.7]\rangle\langle[0.1,0.4],[0.4,0.6]\rangle \\ \langle[0.3,0.5],[0.7,0.8]\rangle\langle[0.2,0.4],[0.1,0.5]\rangle\langle[0.2,0.8],[0.4,0.6]\rangle\langle[0.1,0.3],[0.6,0.9]\rangle\langle[0.3,0.7],[0.1,0.3]\rangle\langle[0.3,0.6],[0.3,0.5]\rangle \\ \langle[0.5,0.7],[0.5,0.6]\rangle\langle[0.2,0.4],[0.4,0.7]\rangle\langle[0.1,0.3],[0.6,0.9]\rangle\langle[0.2,0.3],[0.6,0.9]\rangle\langle[0.3,0.7],[0.2,0.5]\rangle\langle[0.3,0.7],[0.1,0.3]\rangle\end{array}\right)$.

Definition 5. Assume that $(U, \widetilde{E})$ is an IVPFCAS and $\widetilde{E}=$ $\left\{\widetilde{E}_{1}, \widetilde{E}_{2}, \ldots, \widetilde{E}_{n}\right\}$ is an IVPF $\beta$-covering of $U$ for some $\beta=$ $\left\langle\left[s_{L}, s_{U}\right],\left[t_{L}, t_{U}\right]\right\rangle$ and $U=\left\{y_{1}, y_{2}, \ldots, y_{m}\right\}$. Suppose that $\widetilde{N}_{\widetilde{E}}^{\beta}=\left\{\widetilde{N}_{\widetilde{E}(y)}^{\beta} \mid y \in U\right\}$ is an IVPF $\beta$-neighborhood system, where

$$
\begin{aligned}
\widetilde{N}_{\widetilde{E}\left(y_{i}\right)}^{\beta}= & \left\{\left\langley_{j},\left[\mu_{L \tilde{N}_{\widetilde{E}\left(y_{i}\right)}^{\beta}}\left(y_{j}\right), \mu_{U \tilde{N}_{\widetilde{E}}\left(y_{i}\right)}^{\beta}\left(y_{j}\right)\right],\right.\right. \\
& {\left.\left.\left[v_{L \tilde{N}_{\widetilde{E}\left(y_{i}\right)}^{\beta}}\left(y_{j}\right), \nu_{\mathcal{U N}_{\widetilde{E}\left(y_{i}\right)}^{\beta}}\left(y_{j}\right)\right]\right\rangle \mid j=1, \ldots, m\right\}, }
\end{aligned}
$$

for all $i=1, \ldots, m$.

Then, for any $A \in \operatorname{IVPF}(U)$,

$$
\begin{aligned}
A= & \left\{\left\langley_{j},\left[\mu_{L A}\left(y_{j}\right), \mu_{U A}\left(y_{j}\right)\right],\right.\right. \\
& {\left.\left.\left[\nu_{L A}\left(y_{j}\right), v_{U A}\left(y_{j}\right)\right]\right\rangle \mid j=1, \ldots, m\right\}, }
\end{aligned}
$$

and the upper and lower approximations of $A$ with respect to $\widetilde{N} \frac{\beta}{\widetilde{E}}$, denoted by $\widetilde{N} \widetilde{E}(A)$ and $\widetilde{N}_{\widetilde{E}}^{\beta}(A)$, are two IVPFSs; they are defined as follows:

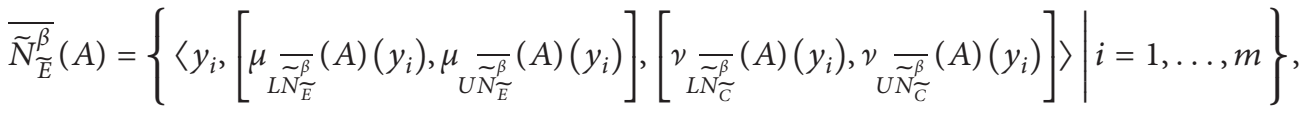

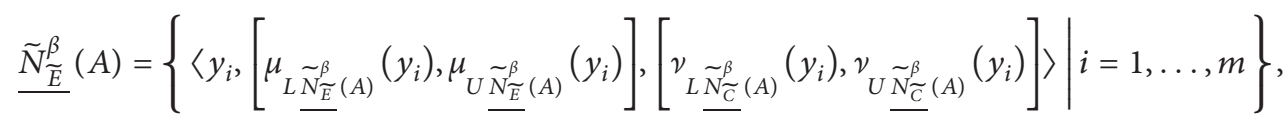

where 


$$
\begin{aligned}
& \mu_{L \overline{\tilde{N}_{\widetilde{E}}^{\beta}}}(A)\left(y_{i}\right)=\bigvee_{j=1}^{m}\left\{\mu_{L \tilde{N}_{\widetilde{E}}^{\beta}\left(y_{i}\right)}\left(y_{j}\right) \wedge \mu_{L A}\left(y_{j}\right)\right\}, \\
& \mu \underset{U \tilde{N}_{\widetilde{E}}^{\beta}(A)}{ }\left(y_{i}\right)=\bigvee_{j=1}^{m}\left\{\mu_{U \widetilde{N}_{\widetilde{E}\left(y_{i}\right)}^{\beta}}\left(y_{j}\right) \wedge \mu_{U A}\left(y_{j}\right)\right\} \text {, } \\
& \nu \underset{L \overline{\tilde{N}}_{\widetilde{E}}^{\beta}(A)}{ }\left(y_{i}\right)=\bigwedge_{j=1}^{m}\left\{\nu_{L \tilde{N}_{\widetilde{E}\left(y_{i}\right)}^{\beta}}\left(y_{j}\right) \vee \nu_{L A}\left(y_{j}\right)\right\}, \\
& v_{\overline{L \tilde{N}_{\widetilde{E}}^{\beta}(A)}}\left(y_{i}\right)=\bigwedge_{j=1}^{m}\left\{v_{L \tilde{N}_{\widetilde{E}}^{\beta}\left(y_{i}\right)}\left(y_{j}\right) \vee v_{L A}\left(y_{j}\right)\right\} \text {, }
\end{aligned}
$$

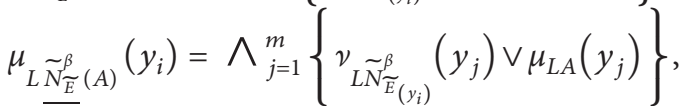

$$
\begin{aligned}
& \mu_{U \tilde{N}_{\widetilde{E}}^{\beta}(A)}^{\beta}\left(y_{i}\right)=\wedge_{j=1}^{m}\left\{v_{U \tilde{N}_{\widetilde{E}\left(y_{i}\right)}^{\beta}}^{\beta}\left(y_{j}\right) \vee \mu_{U A}\left(y_{j}\right)\right\}, \\
& v_{L \tilde{N}_{\widetilde{E}}^{\beta}(A)}\left(y_{i}\right)=\bigvee_{j=1}^{m}\left\{\mu_{L \tilde{N}_{\widetilde{E}}^{\beta}\left(y_{i}\right)}^{\beta}\left(y_{j}\right) \wedge v_{L A}\left(y_{j}\right)\right\},
\end{aligned}
$$

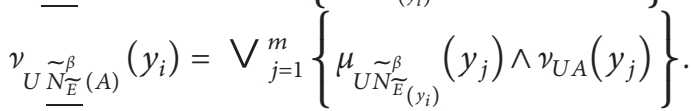

The operators $\overline{\widetilde{N}_{\widetilde{E}}^{\beta}}(A)$ and $\widetilde{N}_{\widetilde{E}}^{\beta}(A)$ are called the upper and lower interval-valued Pythagorean fuzzy rough approximation operators (IVPFRAOs) with respect to $\widetilde{N}_{\widetilde{E}}^{\beta}$, respectively.

Example 2 (continued from Example 1). Let an IVPFS

$$
\begin{aligned}
A= & \left\langle\left\langle y_{1},[0.5,0.7],[0.3,0.4]\right\rangle,\left\langle y_{2},[0.1,0.4],[0.6,0.7]\right\rangle,\right. \\
& \left\langle y_{3},[0.2,0.8],[0.4,0.6]\right\rangle,\left\langle y_{4},[0.6,0.8],[0.1,0.3]\right\rangle, \\
& \left.\left\langle y_{5},[0.3,0.6],[0.3,0.5]\right\rangle,\left\langle y_{6},[0.7,0.9],[0.1,0.2]\right\rangle\right\} .
\end{aligned}
$$

Then,

$\underline{\tilde{N}_{\widetilde{E}}^{\beta}}(A)=\left\{\left\langle y_{1},[0.5,0.7],[0.3,0.4]\right\rangle,\left\langle y_{2},[0.1,0.4],[0.4,0.7]\right\rangle\right.$,

$\left\langle y_{3},[0.2,0.8],[0.4,0.6]\right\rangle,\left\langle y_{4},[0.6,0.8],[0.1,0.3]\right\rangle$,

$\left.\left\langle y_{5},[0.3,0.6],[0.3,0.5]\right\rangle,\left\langle y_{6},[0.7,0.9],[0.1,0.2]\right\rangle\right\}$,

$\overline{\widetilde{N}}_{\widetilde{E}}^{\beta}(A)=\left\{\left\langle y_{1},[0.5,0.7],[0.3,0.4]\right\rangle,\left\langle y_{2},[0.1,0.4],[0.6,0.7]\right\rangle\right.$,

$\left\langle y_{3},[0.2,0.8],[0.4,0.6]\right\rangle,\left\langle y_{4},[0.4,0.8],[0.1,0.3]\right\rangle$,

$\left.\left\langle y_{5},[0.3,0.6],[0.3,0.5]\right\rangle,\left\langle y_{6},[0.5,0.7],[0.2,0.5]\right\rangle\right\}$.

Definition 6. Let $P_{1}=\left\langle\left[\mu_{L A}, \mu_{U A}\right],\left[\nu_{L A}, \nu_{U A}\right]\right\rangle$ and $P_{1}=\left\langle\left[\mu_{L A}, \mu_{U A}\right],\left[\nu_{L A}, \nu_{U A}\right]\right\rangle$ be two IVPFNs, and the generalized distance between $P_{1}$ and $P_{2}$ is defined as follows:

$$
\begin{aligned}
d\left(P_{1}, P_{2}\right)= & \left\{\frac { 1 } { 4 } ( 1 - p ) \left[\left|\mu_{L A}^{2}-\mu_{L B}^{2}\right|^{\lambda}+\left|\mu_{U A}^{2}-\mu_{U B}^{2}\right|^{\lambda}\right.\right. \\
& \left.+\left|v_{L A}^{2}-v_{L B}^{2}\right|^{\lambda}+\left|v_{U A}^{2}-v_{U B}^{2}\right|^{\lambda}\right] \\
& \left.+p\left[\left|\pi_{L A}^{2}-\pi_{L B}^{2}\right|^{\lambda}+\left|\pi_{U A}^{2}-\pi_{U B}^{2}\right|^{\lambda}\right]\right\}^{1 / \lambda},
\end{aligned}
$$

where $\quad \pi_{L A}=\sqrt{1-\mu_{U A}^{2}-\nu_{U A}^{2}}, \quad \pi_{L B}=\sqrt{1-\mu_{U B}^{2}-\nu_{U B}^{2}}$, $\pi_{U A}=\sqrt{1-\mu_{L A}^{2}-v_{L A}^{2}}, \quad \pi_{U B}=\sqrt{1-\mu_{L B}^{2}-v_{L B}^{2}}, \quad \lambda>0$, and $p \in[0,1]$.

When the parameters $\lambda$ and $p$ take different values, we will get some different distance measures.

Case 1. When $\lambda=1$, the distance will be reduced to Hamming-indeterminacy degree-preference distance:

$$
\begin{aligned}
d\left(P_{1}, P_{2}\right)= & \frac{1}{4}(1-p)\left[\left|\mu_{L A}^{2}-\mu_{L B}^{2}\right|+\left|\mu_{U A}^{2}-\mu_{U B}^{2}\right|\right. \\
& \left.+\left|v_{L A}^{2}-v_{L B}^{2}\right|+\left|\nu_{U A}^{2}-\nu_{U B}^{2}\right|\right] \\
& +p\left[\left|\pi_{L A}^{2}-\pi_{L B}^{2}\right|+\left|\pi_{U A}^{2}-\pi_{U B}^{2}\right|\right] .
\end{aligned}
$$

In Case 1, if $p=0$, the effect of the indeterminacy degree is not considered. The distance will be reduced to metric distance:

$$
\begin{aligned}
d\left(P_{1}, P_{2}\right)= & \frac{1}{4}\left[\left|\mu_{L A}^{2}-\mu_{L B}^{2}\right|+\left|\mu_{U A}^{2}-\mu_{U B}^{2}\right|\right. \\
& \left.+\left|\nu_{L A}^{2}-v_{L B}^{2}\right|+\left|\nu_{U A}^{2}-\nu_{U B}^{2}\right|\right]
\end{aligned}
$$

Case 2. When $\lambda=2$, the distance will be reduced to $\mathrm{Eu}-$ clidean-indeterminacy degree-preference distance:

$$
\begin{aligned}
d\left(P_{1}, P_{2}\right)= & \left\{\frac { 1 } { 4 } ( 1 - p ) \left[\left|\mu_{L A}^{2}-\mu_{L B}^{2}\right|^{2}+\left|\mu_{U A}^{2}-\mu_{U B}^{2}\right|^{2}\right.\right. \\
& \left.+\left|v_{L A}^{2}-v_{L B}^{2}\right|^{2}+\left|v_{U A}^{2}-v_{U B}^{2}\right|^{2}\right] \\
& \left.+p\left[\left|\pi_{L A}^{2}-\pi_{L B}^{2}\right|^{2}+\left|\pi_{U A}^{2}-\pi_{U B}^{2}\right|^{2}\right]\right\}^{1 / 2} .
\end{aligned}
$$

In Case 2, if $p=0$, the distance will be reduced to Euclidean distance:

$$
\begin{aligned}
d\left(P_{1}, P_{2}\right)= & \left\{\frac { 1 } { 4 } \left[\left|\mu_{L A}^{2}-\mu_{L B}^{2}\right|^{2}+\left|\mu_{U A}^{2}-\mu_{U B}^{2}\right|^{2}\right.\right. \\
& \left.\left.+\left|\nu_{L A}^{2}-\nu_{L B}^{2}\right|^{2}+\left|\nu_{U A}^{2}-\nu_{U B}^{2}\right|^{2}\right]\right\}^{1 / 2} .
\end{aligned}
$$

Let $P_{1}$ and $P_{2}$ be two IVPFNs, and it is easy to verify that the distance $d$ satisfied the following properties:

(1) $d\left(P_{1}, P_{2}\right) \geq 0$

(2) $d\left(P_{1}, P_{2}\right)=d\left(P_{2}, P_{1}\right)$

(3) $d\left(P_{1}, P_{2}\right)=0 \Longleftrightarrow A=B$ 
Definition 7. Let $A$ and $B$ be two IVPFSs. The distance $D(A, B)$ of $A$ and $B$. is defined as follows:

$$
\begin{aligned}
D(A, B)= & \frac{1}{|U|} \sum_{y \in U}\left\{\frac { 1 } { 4 } ( 1 - p ) \left[\left|\mu_{L A}^{2}(y)-\mu_{L B}^{2}(y)\right|^{\lambda}\right.\right. \\
& +\left|\mu_{U A}^{2}(y)-\mu_{U B}^{2}(y)\right|^{\lambda}+\left|\nu_{L A}^{2}(y)-v_{L B}^{2}(y)\right|^{\lambda} \\
& \left.+\left|\nu_{U A}^{2}(y)-v_{U B}^{2}(y)\right|^{\lambda}\right] \\
& \left.+p\left[\left|\pi_{L A}^{2}(y)-\pi_{L B}^{2}(y)\right|^{\lambda}+\left|\pi_{U A}^{2}(y)-\pi_{U B}^{2}(y)\right|^{\lambda}\right]\right\}^{1 / \lambda},
\end{aligned}
$$

where $\lambda>0$ and $p \in[0,1]$.

Definition 8. Suppose that $(U, \widetilde{E})$ is an IVPFCAS and $\widetilde{E}=$ $\left\{\widetilde{E}_{1}, \widetilde{E}_{2}, \ldots, \widetilde{E}_{n}\right\}$ is an IVPF $\beta$-covering of $U$ for some $\beta=\left\langle\left[s_{L}, s_{U}\right],\left[t_{L}, t_{U}\right]\right\rangle$. Suppose that $\widetilde{N}_{\widetilde{E}}^{\beta}=\left\{\widetilde{N}_{\widetilde{E}(y)}^{\beta} \mid y \in U\right\}$ is an IVPF $\beta$-neighborhood system. For any $A \in \operatorname{IVPF}(U)$, $\widetilde{N}_{\widetilde{E}}^{\beta}(A)$ and $\overline{\widetilde{N}_{\widetilde{E}}^{\beta}}(A)$ are the lower and upper approximations $\overline{\text { of } A}$. The rough degree and precision degree of $A$ are defined as follows:

$$
\begin{aligned}
& R_{\widetilde{N}_{E}^{\beta}(A)}=D\left(\underline{\widetilde{N_{\widetilde{E}}^{\beta}}}(A), \overline{\tilde{N}_{\widetilde{E}}^{\beta}}(A)\right), \\
& P_{\underset{\mathcal{N}^{\beta}}{\sim}(A)}=1-R_{\underset{\mathcal{N}^{\sim}}{\sim}(A)} .
\end{aligned}
$$

Example 3. (continued from Example 2). Let $\lambda=4$ and $p=0.5$. According to Definitions 6 and 7 , the rough degree and precision degree of $A$ with respect to $\widetilde{N}_{\widetilde{E}}^{\beta}$ can be calculated as follows:

$$
\begin{aligned}
& R_{\underset{\sim^{N}}{\sim}(A)}=0.1188, \\
& P_{\underset{\sim_{N}^{\beta}}{\sim}(A)}=0.8812 .
\end{aligned}
$$

\section{A Novel Method to Solve MADM Problems with IVPFI}

In Section 3, we established the CIVPFRS. Based on this, we will construct a model and method for a class of MADM problems with IVPFI in this section. In addition, we will propose a decision-making algorithm to solve the MADM problems.

4.1. Decision-Making Method. We will propose the model and method, which is used to solve the MADM problems with IVPFI based on CIVPFRS.

4.1.1. The Form Descriptions of Problem of MADM Problem with IVPFI. Assume that $U=\left\{y_{1}, y_{2}, \ldots, y_{m}\right\}$ is a set of alternatives and $\widetilde{E}=\left\{\widetilde{E}_{1}, \widetilde{E}_{2}, \ldots, \widetilde{E}_{n}\right\}$ is the set of attributes.
Let $W=\left(w_{1}, w_{2}, \ldots, w_{n}\right)^{T}$ be the weight vector of the attributes, where $0 \leq w_{j} \leq 1, j=1,2, \ldots, n$, which satisfies $\sum_{j=1}^{n} w_{j}=1$. Let the decision maker $D$ present the evaluation values of the attributes $y_{i}, i=1, \ldots, m$ with respect to the attribute set $\widetilde{E}_{j}(j=1, \ldots, n)$ by $\widetilde{E}_{j}\left(y_{i}\right)=\left\langle\left[\mu_{L}, \mu_{U}\right]\right.$, $\left.\left[\nu_{L}, v_{U}\right]\right\rangle_{i j}$. It means, the MD to which $y_{i}$ satisfies $\widetilde{E}_{j}$ is interval-valued $\left[\mu_{L}, \mu_{U}\right]$, and the NMD to which $y_{i}$ dissatisfies $\widetilde{E}_{j}$ is interval-valued $\left[\nu_{L}, v_{U}\right]$. By using IVPF-TOPSIS method, we establish IVPF-PIS $\operatorname{IVP}^{+}=\left\{\widetilde{E}_{j}, \max \left\{s\left(\widetilde{E}_{j}\right.\right.\right.$ $\left.\left.\left.\left(y_{i}\right)\right)\right\} \mid j=1, \ldots, n\right\}$ and IVPF-NIS $\operatorname{IVP}^{-}=\left\{\widetilde{E}_{j}, \min \left\{s\left(\widetilde{E}_{j}\right.\right.\right.$ $\left.\left.\left.\left(y_{i}\right)\right)\right\} \mid j=1, \ldots, n\right\}$, where $s$ is the score function, which is defined in Definition 3. After that we calculate the distances $D^{+}$and $D^{-}$between each alternative $y_{i}$ and $\mathrm{IVP}^{+}$and $\mathrm{IVP}^{-}$, respectively, according to Definition 6. Therefore, we construct a new IVPFS $D=\left\langle\left[\mu_{D L}, \mu_{D U}\right],\left[\nu_{D L}, v_{D U}\right]\right\rangle=\left\langle\left[D^{+}\right.\right.$, $\left.\left.D^{+}\right],\left[D^{-}, D^{-}\right]\right\rangle$.

In this section, a new method is proposed to solve the MADM problem by using the CIVPFRS established in Section 3.

4.1.2. Decision-Making Method and Process. Considering the characteristics of MADM problems, we will present a decision-making model and its algorithm steps based on CIVPFRS. This model consists of three parts. In the first part, we will determine IVPF decision objectives for all alternatives. In the second part, we will use the precision parameter $\beta(0<\beta \leq 1)$ to calculate the upper and lower approximations of the IVPF decision-making object for all alternatives. In the third part, on the basis of the first two steps, we use the decision-making principle to rank all alternatives and then give the optimal object of the MADM problem.

Next, we will introduce in detail the model and method for solving the MADM problem based on CIVPFRS.

First, we will propose the IVPF-TOPSIS method. This method takes into account the following principles. The optimal alternative should be the one with the shortest distance from IVPF-PIS and the longest distance from IVPF-NIS.

IVPF-PIS and IVPF-NIS are calculated using the score function, which is defined in Definition 3:

$$
\begin{aligned}
\operatorname{IVP}^{+}= & \left\{\widetilde{E}_{j}, \max \left\{s\left(\widetilde{E}_{j}\left(y_{i}\right)\right)\right\} \mid j=1, \ldots, n\right\}(i=1, \ldots, m) \\
= & \left\{\left\langle\widetilde{E}_{1},\left[\mu_{1 L}^{+}, \mu_{1 U}^{+}\right],\left[\nu_{1 L}^{+}, v_{1 U}^{+}\right]\right\rangle, \ldots,\left\langle\widetilde{E}_{n},\left[\mu_{n L}^{+}, \mu_{n U}^{+}\right],\right.\right. \\
& {\left.\left.\left[v_{n L}^{+}, v_{n U}^{+}\right]\right\rangle\right\}, }
\end{aligned}
$$

$$
\begin{aligned}
\operatorname{IVP}^{-}= & \left\{\widetilde{E}_{j}, \min \left\{s\left(\widetilde{E}_{j}\left(y_{i}\right)\right)\right\} \mid j=1, \ldots, n\right\}(i=1, \ldots, m) \\
= & \left\{\left\langle\widetilde{E}_{1},\left[\mu_{1 L}^{-}, \mu_{1 U}^{-}\right],\left[\nu_{1 L}^{-}, \nu_{1 U}^{-}\right]\right\rangle, \ldots,\left\langle\widetilde{E}_{n},\left[\mu_{n L}^{-}, \mu_{n U}^{-}\right],\right.\right. \\
& {\left.\left.\left[\nu_{n L}^{-}, \nu_{n U}^{-}\right]\right\rangle\right\} . }
\end{aligned}
$$

Next, according to Definition 6 , we calculate $D^{+}$and $D^{-}$ between each alternative $y_{i}$ and $\mathrm{IVP}^{+}$and $\mathrm{IVP}^{-}$, respectively, for all $i=1,2, \ldots, m$, as follows: 


$$
\begin{aligned}
D^{+}= & \sum_{j=1}^{n} w_{j} \cdot d\left(\left(\widetilde{E}_{j}\left(y_{i}\right)\right),\left(\widetilde{E}_{j}\left(\mathrm{IVP}^{+}\right)\right)\right) \\
= & \sum_{j=1}^{n} w_{j} \cdot\left\{\frac{1}{4}(1-p)\left[\left|\mu_{L_{i j}}^{2}-\mu_{j L}^{+2}\right|^{\lambda}+\left|\mu_{U_{i j}}^{2}-\mu_{j U}^{+2}\right|^{\lambda}+\left|v_{L_{i j}}^{2}-v_{j L}^{+2}\right|^{\lambda}+\left|\nu_{U_{i j}}^{2}-v_{j U}^{+2}\right|^{\lambda}\right]\right. \\
& \left.+p\left[\left|\pi_{L_{i j}}^{2}-\pi_{j L}^{+2}\right|^{\lambda}+\left|\pi_{U_{i j}}^{2}-\pi_{j U}^{+2}\right|^{\lambda}\right]\right\}^{1 / \lambda}, \\
D^{-}= & \sum_{j=1}^{n} w_{j} \cdot d\left(\left(\widetilde{E}_{j}\left(y_{i}\right)\right),\left(\widetilde{E}_{j}\left(\mathrm{IVP}^{-}\right)\right)\right) \\
= & \sum_{j=1}^{n} w_{j} \cdot\left\{\frac { 1 } { 4 } ( 1 - p ) \left[\left|\mu_{L_{i j}}^{2}-\mu_{j L}^{-2}\right|^{\lambda}+\left|\mu_{U_{i j}}^{2}-\mu_{j U}^{-2}\right|^{\lambda}+\left|\nu_{L_{i j}}^{2}-v_{j L}^{-2}\right|^{\lambda}\right.\right. \\
& \left.\left.+\left|\nu_{U_{i j}}^{2}-v_{j U}^{-2}\right|^{\lambda}\right]+p\left[\left|\pi_{L_{i j}}^{2}-\pi_{j L}^{-2}\right|^{\lambda}+\left|\pi_{U_{i j}}^{2}-\pi_{j U}^{-2}\right|^{\lambda}\right]\right\}^{1 / \lambda} \cdot
\end{aligned}
$$

Here, we construct a new IVPFS $D=\left\langle\left[\mu_{D L}, \mu_{D U}\right]\right.$, $\left.\left[\nu_{D L}, \nu_{D U}\right]\right\rangle=\left\langle\left[D^{+}, D^{+}\right],\left[D^{-}, D^{-}\right]\right\rangle$. In order to fuse all preference information in the framework of CIVPFRS, we introduced the fuzzy logical operators [35] and then propose another method to fuse fuzzy preference information of MADM problems.

Triangular norms ( $t$-norms) were first proposed in the framework of probabilistic metric spaces [35]. In some contexts, $t$-norms have been proved to be an appropriate tool for information fusion of the upper and lower approximations of interval-valued Pythagorean fuzzy decisionmaking object.

Definition 9 (see [35]). t-norm is an associative, increasing and commutative mapping function $\mathscr{T}:[0,1]^{2} \longrightarrow[0,1]$, which satisfies the boundary condition, for any $(x \in[0,1], \mathscr{T}(x, 1)=x)$. Moreover, the triangular conorm ( $t$-conorm) is an associative, increasing and commutative mapping function $\delta:[0,1]^{2} \longrightarrow[0,1]$, which satisfies the boundary condition, for any $(x \in[0,1], \mathcal{S}(x, 0)=x)$.

In the current work, we consider the following the $t$-norms and $t$-conorms for MADM problems:

$$
\begin{aligned}
& \mathscr{T}_{P}(x, y)=\frac{x y}{\sqrt{1+\left(1-x^{2}\right)\left(1-y^{2}\right)}}, \\
& \mathcal{S}_{P}(x, y)=\sqrt{\frac{\left(x^{2}+y^{2}\right)}{\left(1+x^{2} y^{2}\right)}} .
\end{aligned}
$$

Secondly, based on the precision parameter $\beta$, we calculate the upper and lower approximations of the best and worst interval-valued Pythagorean fuzzy decision-making objects by Definition 5, respectively:

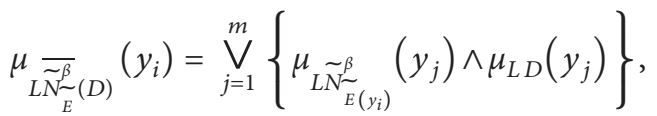

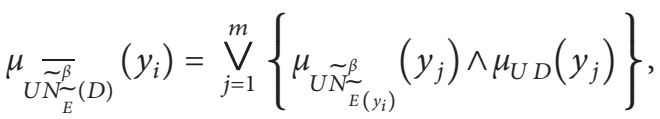

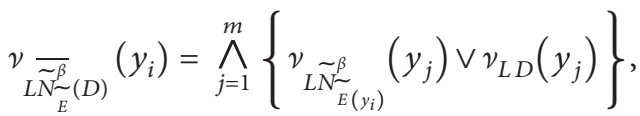

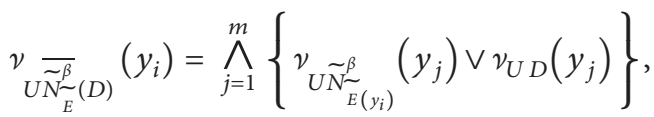

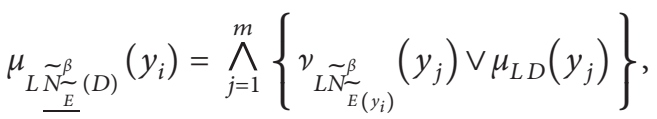

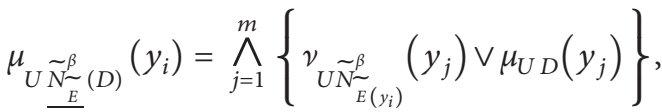

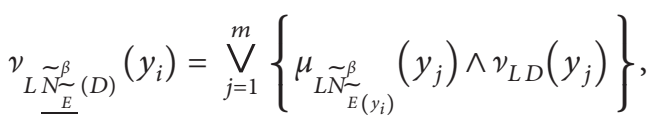

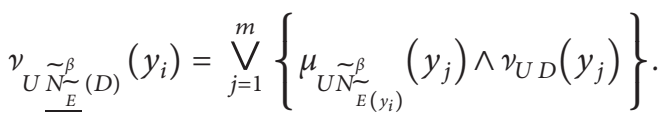

Then, according to the upper and lower approximations of the IVPF decision-making object with the consistency consensus threshold $\beta$, we define a ranking function to solve the MADM problem.

Definition 10. For the IVPF decision-making object, $D=\left\langle\left[D^{+}, D^{+}\right],\left[D^{-}, D^{-}\right]\right\rangle \in \operatorname{IVPF}(U)$, and the preference threshold is $\alpha(0<\alpha \leq 1)$. The ranking functions of the lower and upper boundaries of MD and NMD of each alternative $y_{i},(i=1, \ldots, m)$ with respect to $\operatorname{IVPF}(U)$ are defined as follows, respectively: 


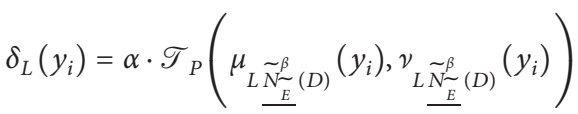

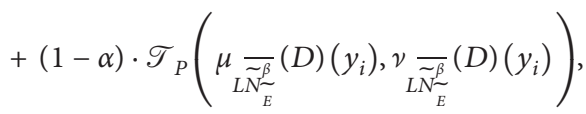

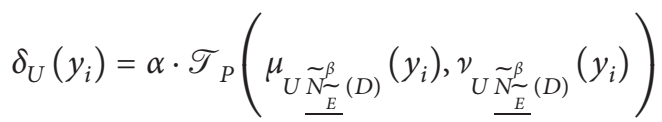

$$
\begin{aligned}
& +(1-\alpha) \cdot \mathscr{T}_{P}\left(\mu \underset{U \bar{N}_{E}^{-\beta}}{\overline{\sigma^{\alpha}}}(D)\left(y_{i}\right), v_{U \bar{N}_{E}^{-\beta}}(D)\left(y_{i}\right)\right) \text {. }
\end{aligned}
$$

With the definitions of ranking functions of the MADM problem, the following conclusion is obvious, that is,

$$
\begin{aligned}
0 & \leq \delta_{L}\left(y_{i}\right), \\
\delta_{U}\left(y_{i}\right) & \leq 1, \\
\forall y_{i} & \in U, \\
i & =1, \ldots, m .
\end{aligned}
$$

Finally, we construct the ranking function of $\delta(y)$ for all $y \in U:$

$$
\delta\left(y_{i}\right)=\frac{1}{2}\left(\delta_{L}\left(y_{i}\right)+\delta_{U}\left(y_{i}\right)\right) \quad(i=1,2, \ldots, m) .
$$

Therefore, we can use the ranking function $\delta(y)$ to get the final optimal object of all $y \in U$ for MADM problems.

4.2. Algorithm for the MADM. In this section, we present the algorithm flow of solving the MADM problem based on CIVPFRS as shown in Algorithm 1.

\section{An Illustrative Example}

Electronic health records (EHRs) technology is beneficial to medical service providers because it can improve service levels and ensure better medical quality. However, the wide variety of open-source EHRs systems makes it difficult for the hospital administrator to decide which system to use. In Ref [36], six popular and active open-source EHRs systems were selected: $U=\left\{\right.$ FreeMED $\left(y_{1}\right)$, GNUmed $\left(y_{2}\right)$, GNU Health $\left(y_{3}\right)$, Hospital $\left(y_{4}\right), \operatorname{HOSxP}\left(y_{5}\right)$, and OpenEMR $\left.\left(y_{6}\right)\right\}$.

The set of evaluation criteria is $\widetilde{E}=\left\{\operatorname{Usability}\left(\widetilde{E_{1}}\right)\right.$, Functionality and features $\left(\widetilde{E}_{2}\right)$, Customisation $\left(\widetilde{E}_{3}\right)$, Ease of installation $\left(\widetilde{E_{4}}\right)$, Risk $\left(\widetilde{E}_{5}\right)$, and Staff Training $\left.\left(\widetilde{E}_{6}\right)\right\}$ (please refer to Ref [36] for detailed description and definition of attribute set).

Due to the uncertainty, imprecision, and hesitancy of the selection problem, decision maker uses IVPFNs to evaluate various open-source EHRs systems. The evaluation results are shown in Table 3. The weight information of each evaluation criteria is as follows: $w_{1}=0.24, w_{2}=0.20, w_{3}=$ $0.15, w_{4}=0.10, w_{5}=0.18$, and $w_{6}=0.13$.

Here, we will use the method based on CIVPFRS to solve the problem of selecting open-source EHRs systems in hospitals. The selection problem of hospital open-source EHRs systems is cited from [36] and makes some improvement under the environment of MADM. Then, we will illustrate the principle and steps of the model and IVPFTOPSIS in solving the MADM problem through the example.

We calculate IVPF-PIS IVP ${ }^{+}$and IVPF-NIS IVP $^{-}$as follows, by using formulas (25) and (26):

$$
\begin{aligned}
\operatorname{IVP}^{+}= & \left\{\left\langle\widetilde{E}_{1},[0.5,0.8],[0.4,0.5]\right\rangle,\left\langle\widetilde{E}_{2},[0.8,0.9],[0.2,0.4]\right\rangle,\left\langle\widetilde{E}_{3},[0.8,0.9],[0.2,0.3]\right\rangle,\right. \\
& \left.\left\langle\widetilde{E}_{4},[0.8,0.9],[0.4,0.4]\right\rangle,\left\langle\widetilde{E}_{5},[0.6,0.9],[0.2,0.4]\right\rangle,\left\langle\widetilde{E}_{6},[0.7,0.9],[0.1,0.3]\right\rangle\right\}, \\
\operatorname{IVP}^{-}= & \left\{\left\langle\widetilde{E}_{1},[0.2,0.3],[0.8,0.9]\right\rangle,\left\langle\widetilde{E}_{2},[0.2,0.5],[0.7,0.8]\right\rangle,\left\langle\widetilde{E}_{3},[0.1,0.2],[0.5,0.8]\right\rangle,\right. \\
& \left.\left\langle\widetilde{E}_{4},[0.3,0.4],[0.7,0.8]\right\rangle,\left\langle\widetilde{E}_{5},[0.1,0.3],[0.5,0.6]\right\rangle,\left\langle\widetilde{E}_{6},[0.2,0.3],[0.8,0.9]\right\rangle\right\} .
\end{aligned}
$$

Then, we calculate the distances $\mu_{D}=D^{+}$and $\nu_{D}=D^{-}$, according to formulas (27) and (28). Let $\lambda=120$ and $p=0.5$. The calculation results are shown in Table 4.

Let the threshold IVPFN be $\beta=\langle[0.1,0.2],[0.5,0.8]\rangle\}$; therefore,

$$
\begin{aligned}
& \widetilde{N}_{\widetilde{E}\left(y_{1}\right)}^{\beta}=\widetilde{E}_{2} \cap \widetilde{E}_{3} \cap \widetilde{E}_{4} \cap \widetilde{E}_{5} \cap \widetilde{E}_{6}, \\
& \widetilde{N}_{\widetilde{E}\left(y_{2}\right)}^{\beta}=\widetilde{E}_{1} \cap \widetilde{E}_{2} \cap \widetilde{E}_{3} \cap \widetilde{E}_{4} \cap \widetilde{E}_{5} \cap \widetilde{E}_{6}, \\
& \widetilde{N}_{\widetilde{E}\left(y_{3}\right)}^{\beta}=\widetilde{E}_{2} \cap \widetilde{E}_{3} \cap \widetilde{E}_{4} \cap \widetilde{E}_{5} \cap \widetilde{E}_{6},
\end{aligned}
$$

$$
\begin{aligned}
& \widetilde{N}_{\widetilde{E}\left(y_{4}\right)}^{\beta}=\widetilde{E}_{1} \cap \widetilde{E}_{3} \cap \widetilde{E}_{5} \cap \widetilde{E}_{6}, \\
& \widetilde{N}_{\widetilde{E}_{\left(y_{5}\right)}^{\beta}}^{\beta}=\widetilde{E}_{2} \cap \widetilde{E}_{3} \cap \widetilde{E}_{4} \cap \widetilde{E}_{5}, \\
& \widetilde{N}_{\widetilde{E}_{\left(y_{6}\right)}^{\beta}}^{\beta}=\widetilde{E}_{1} \cap \widetilde{E}_{2} \cap \widetilde{E}_{3} \cap \widetilde{E}_{5} \cap \widetilde{E}_{6} .
\end{aligned}
$$

By calculation, we can obtain Table 5 .

We calculate the lower and upper approximations, according to formulas (12) and (13). The results are shown in Table 6. 
Input IVPFCAS $(U, \widetilde{E})$;

Output Ranking results of all alternatives;

Step 1. Construct IVPF-PIS IVP ${ }^{+}$and IVPF-NIS IVP $^{-}$by formulas (25) and (26);

Step 2. Calculate the distances $\mu_{D}=D^{+}$and $\nu_{D}=D^{-}$by using formulas (27) and (28), respectively.

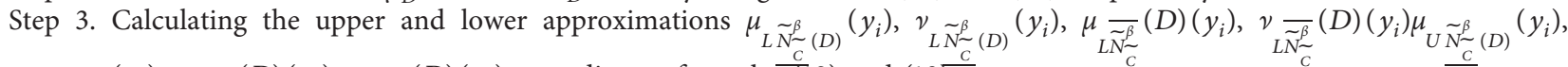

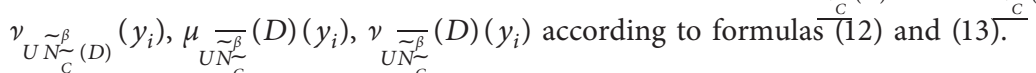

Step 4 . Determine risk preference threshold $\alpha(0<\alpha \leq 1)$ and calculate those ranking functions $\delta_{L}\left(y_{i}\right)$ and $\delta_{U}\left(y_{i}\right)$ by using formulas (31) and (32).

Step 5. Calculate the ranking function $\delta\left(y_{i}\right)$ according to formula (34).

Step 6. List the ranking results of all alternatives.

Algorithm 1: Algorithm for MADM with CIVPFRS.

TABLE 3: IVPF $\beta$-covering $\widetilde{E}$.

\begin{tabular}{lcccccc}
\hline$U / \widetilde{E}$ & $\widetilde{E}_{1}$ & $\widetilde{E}_{2}$ & $\widetilde{E}_{3}$ & $\widetilde{E}_{4}$ & $\widetilde{E}_{5}$ & $\widetilde{E}_{6}$ \\
\hline$y_{1}$ & $\langle[0.2,0.3],[0.8,0.9]\rangle$ & $\langle[0.5,0.7],[0.3,0.4]\rangle$ & $\langle[0.4,0.5],[0.5,0.8]\rangle$ & $\langle[0.2,0.5],[0.5,0.8]\rangle$ & $\langle[0.6,0.9],[0.2,0.4]\rangle$ & $\langle[0.7,0.9],[0.1,0.3]\rangle$ \\
$y_{2}$ & $\langle[0.6,0.7],[0.3,0.8]\rangle$ & $\langle[0.4,0.6],[0.1,0.5]\rangle$ & $\langle[0.4,0.6],[0.3,0.5]\rangle$ & $\langle[0.8,0.9],[0.4,0.4]\rangle$ & $\langle[0.1,0.3],[0.5,0.6]\rangle$ & $\langle[0.4,0.5],[0.5,0.5]\rangle$ \\
$y_{3}$ & $\langle[0.4,0.5],[0.6,0.7]\rangle$ & $\langle[0.8,0.9],[0.2,0.4]\rangle$ & $\langle[0.7,0.9],[0.3,0.4]\rangle$ & $\langle[0.5,0.6],[0.1,0.2]\rangle$ & $\langle[0.6,0.7],[0.2,0.3]\rangle$ & $\langle[0.6,0.8],[0.2,0.4]\rangle$ \\
$y_{4}$ & $\langle[0.4,0.6],[0.1,0.3]\rangle$ & $\langle[0.2,0.5],[0.7,0.8]\rangle$ & $\langle[0.1,0.2],[0.5,0.8]\rangle$ & $\langle[0.3,0.4],[0.7,0.8]\rangle$ & $\langle[0.4,0.7],[0.1,0.4]\rangle$ & $\langle[0.4,0.7],[0.1,0.5]\rangle$ \\
$y_{5}$ & $\langle[0.2,0.3],[0.6,0.8]\rangle$ & $\langle[0.6,0.7],[0.4,0.5]\rangle$ & $\langle[0.6,0.7],[0.4,0.5]\rangle$ & $\langle[0.6,0.8],[0.3,0.5]\rangle$ & $\langle[0.6,0.8],[0.4,0.5]\rangle$ & $\langle[0.2,0.3],[0.8,0.9]\rangle$ \\
$y_{6}$ & $\langle[0.5,0.8],[0.4,0.5]\rangle$ & $\langle[0.7,0.5],[0.1,0.5]\rangle$ & $\langle[0.8,0.9],[0.2,0.3]\rangle$ & $\langle[0.2,0.5],[0.7,0.8]\rangle$ & $\langle[0.7,0.8],[0.2,0.4]\rangle$ & $\langle[0.5,0.6],[0.4,0.5]\rangle$ \\
\hline
\end{tabular}

TABLE 4: Calculation results of distance $D^{+}$and $D^{-}$.

\begin{tabular}{lcccccc}
\hline$U$ & $y_{1}$ & $y_{2}$ & $y_{3}$ & $y_{4}$ & $y_{5}$ & $y_{6}$ \\
\hline$D^{+}$ & 0.3505 & 0.4631 & 0.2623 & 0.5004 & 0.3972 & 0.2570 \\
$D^{-}$ & 0.3745 & 0.4170 & 0.5197 & 0.3211 & 0.3544 & 0.5179 \\
\hline
\end{tabular}

TABLE 5: Tabular representation of $\widetilde{N}_{\tilde{E}}^{\beta}$.

\begin{tabular}{ccccccc}
\hline$\tilde{N}_{\tilde{E}}^{\beta}$ & $y_{1}$ & $y_{2}$ & $y_{3}$ & $y_{4}$ & $y_{5}$ & $y_{6}$ \\
\hline$y_{1}$ & $\langle[0.2,0.5],[0.5,0.8]\rangle$ & $\langle[0.1,0.3],[0.5,0.6]\rangle$ & $\langle[0.5,0.6],[0.3,0.4]\rangle$ & $\langle[0.1,0.2],[0.7,0.8]\rangle$ & $\langle[0.2,0.3],[0.8,0.9]\rangle$ & $\langle[0.2,0.5],[0.7,0.8]\rangle$ \\
$y_{2}$ & $\langle[0.2,0.3],[0.8,0.9]\rangle$ & $\langle[0.1,0.3],[0.5,0.8]\rangle$ & $\langle[0.4,0.5],[0.6,0.7]\rangle$ & $\langle[0.1,0.2],[0.7,0.8]\rangle$ & $\langle[0.2,0.3],[0.8,0.9]\rangle$ & $\langle[0.2,0.5],[0.7,0.8]\rangle$ \\
$y_{3}$ & $\langle[0.2,0.5],[0.5,0.8]\rangle$ & $\langle[0.1,0.3],[0.5,0.6]\rangle$ & $\langle[0.5,0.6],[0.3,0.4]\rangle$ & $\langle[0.1,0.2],[0.7,0.8]\rangle$ & $\langle[0.2,0.3],[0.8,0.9]\rangle$ & $\langle[0.2,0.5],[0.7,0.8]\rangle$ \\
$y_{4}$ & $\langle[0.2,0.3],[0.8,0.9]\rangle$ & $\langle[0.1,0.3],[0.5,0.8]\rangle$ & $\langle[0.4,0.5],[0.6,0.7]\rangle$ & $\langle[0.1,0.2],[0.5,0.8]\rangle$ & $\langle[0.2,0.3],[0.8,0.9]\rangle$ & $\langle[0.5,0.6],[0.4,0.5]\rangle$ \\
$y_{5}$ & $\langle[0.2,0.5],[0.5,0.8]\rangle$ & $\langle[0.1,0.3],[0.5,0.6]\rangle$ & $\langle[0.5,0.6],[0.3,0.4]\rangle$ & $\langle[0.1,0.2],[0.7,0.8]\rangle$ & $\langle[0.6,0.7],[0.4,0.5]\rangle$ & $\langle[0.2,0.5],[0.7,0.8]\rangle$ \\
$y_{6}$ & $\langle[0.2,0.3],[0.8,0.9]\rangle$ & $\langle[0.1,0.3],[0.5,0.8]\rangle$ & $\langle[0.4,0.5],[0.6,0.7]\rangle$ & $\langle[0.1,0.2],[0.7,0.8]\rangle$ & $\langle[0.2,0.3],[0.8,0.9]\rangle$ & $\langle[0.5,0.5],[0.4,0.5]\rangle$ \\
\hline
\end{tabular}

TABLE 6: The lower and upper approximations.

\begin{tabular}{|c|c|c|c|c|c|c|}
\hline$U$ & $y_{1}$ & $y_{2}$ & $y_{3}$ & $y_{4}$ & $y_{5}$ & $y_{6}$ \\
\hline$\left.\mu_{L} \widetilde{N}_{E}^{\beta}(D)\right)$ & 0.3 & 0.5 & 0.3 & 0.4 & 0.3 & 0.4 \\
\hline 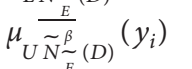 & 0.4 & 0.7 & 0.4 & 0.5 & 0.4 & 0.5 \\
\hline 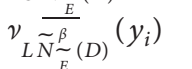 & 0.5 & 0.4 & 0.5 & 0.5 & 0.5 & 0.5 \\
\hline$\nu_{U \sim \tilde{N}^{\beta} \sim} \frac{E}{\tilde{F}_{(}^{\beta}(D)}\left(y_{i}\right)$ & 0.5197 & 0.5 & 0.5197 & 0.5179 & 0.5197 & 0.5 \\
\hline$\mu \underset{L \tilde{N}^{\beta}}{\frac{E}{\bar{\beta}^{\beta}}}(D)\left(y_{i}\right)$ & 0.2623 & 0.2623 & 0.2623 & 0.2623 & 0.3972 & 0.2623 \\
\hline$\mu \underset{U N \tilde{N} \sim}{\frac{E}{\beta}}(D)\left(y_{i}\right)$ & 0.3505 & 0.3 & 0.3505 & 0.3 & 0.3972 & 0.3 \\
\hline$v \underset{L \tilde{N}_{E}^{\beta}}{\frac{E}{\tilde{\beta}}}(D)\left(y_{i}\right)$ & 0.5 & 0.5 & 0.5 & 0.5 & 0.4 & 0.5 \\
\hline$\nu \underset{U \tilde{N}_{E}^{\beta}}{\bar{\sim}}(D)\left(y_{i}\right)$ & 0.5197 & 0.7 & 0.5197 & 0.5179 & 0.5 & 0.5179 \\
\hline
\end{tabular}

TABLE 7: The result of ranking functions.

\begin{tabular}{lcccccc}
\hline$U$ & $y_{1}$ & $y_{2}$ & $y_{3}$ & $y_{4}$ & $y_{5}$ & $y_{6}$ \\
\hline$\delta_{L}\left(y_{i}\right)$ & 0.1119 & 0.1426 & 0.1119 & 0.1426 & 0.1171 & 0.1426 \\
$\delta_{U}\left(y_{i}\right)$ & 0.1583 & 0.2666 & 0.1583 & 0.1861 & 0.1616 & 0.1801 \\
$\delta\left(y_{i}\right)$ & 0.1351 & 0.2046 & 0.1351 & 0.1644 & 0.1394 & 0.1614 \\
\hline
\end{tabular}

Consider the risk preference threshold $\alpha=0.75$. Then, according to equations (31)-(34), we calculate the ranking functions. The results are shown in Table 7 .

Finally, based on the values of the ranking function $\delta\left(y_{i}\right)(i=1,2, \ldots, 6)$, we present the optimal ranking of all hospital open-source EHRs systems as follows:

$$
y_{2}>y_{4}>y_{6}>y_{5}>y_{3}=y_{1} \text {. }
$$


TABLE 8: The effect of parameter $\lambda$ changes.

\begin{tabular}{lll}
\hline Parameter & \multicolumn{1}{c}{ Ordering index of $y_{i}(i=1,2, \ldots, 6)$} & Ranking order \\
\hline$\lambda=1$ & $\delta\left(y_{1}\right)=0.0985, \delta\left(y_{2}\right)=0.1783, \delta\left(y_{3}\right)=0.0985, \delta\left(y_{4}\right)=0.1297, \delta\left(y_{5}\right)=0.1007, \delta\left(y_{6}\right)=0.1297$ & $y_{2}>y_{4}=y_{6}>y_{5}>y_{3}=y_{1}$ \\
$\lambda=2$ & $\delta\left(y_{1}\right)=0.0917, \delta\left(y_{2}\right)=0.1673, \delta\left(y_{3}\right)=0.0917, \delta\left(y_{4}\right)=0.1222, \delta\left(y_{5}\right)=0.0939, \delta\left(y_{6}\right)=0.1222$ & $y_{2}>y_{4}=y_{6}>y_{5}>y_{3}=y_{1}$ \\
$\lambda=12$ & $\delta\left(y_{1}\right)=0.1161, \delta\left(y_{2}\right)=0.1933, \delta\left(y_{3}\right)=0.1161, \delta\left(y_{4}\right)=0.1460, \delta\left(y_{5}\right)=0.1206, \delta\left(y_{6}\right)=0.1460$ & $y_{2}>y_{4}=y_{6}>y_{5}>y_{3}=y_{1}$ \\
$\lambda=120$ & $\delta\left(y_{1}\right)=0.1351, \delta\left(y_{2}\right)=0.2046, \delta\left(y_{3}\right)=0.1351, \delta\left(y_{4}\right)=0.1644, \delta\left(y_{5}\right)=0.1394, \delta\left(y_{6}\right)=0.1614$ & $y_{2}>y_{4}>y_{6}>y_{5}>y_{3}=y_{1}$ \\
$\lambda=1200$ & $\delta\left(y_{1}\right)=0.0980, \delta\left(y_{2}\right)=0.1778, \delta\left(y_{3}\right)=0.0980, \delta\left(y_{4}\right)=0.1318, \delta\left(y_{5}\right)=0.0989, \delta\left(y_{6}\right)=0.1318$ & $y_{2}>y_{4}=y_{6}>y_{5}>y_{3}=y_{1}$ \\
\hline
\end{tabular}

Table 9: The effect of parameter $p$ changes.

\begin{tabular}{llc}
\hline Parameter & Ordering index of $y_{i}(i=1,2, \ldots, 6)$ & Ranking order \\
\hline$p=0.1$ & $\delta\left(y_{1}\right)=0.1356, \delta\left(y_{2}\right)=0.2046, \delta\left(y_{3}\right)=0.1356, \delta\left(y_{4}\right)=0.1648, \delta\left(y_{5}\right)=0.1398, \delta\left(y_{6}\right)=0.1614$ & $y_{2}>y_{4}>y_{6}>y_{5}>y_{3}=y_{1}$ \\
$p=0.3$ & $\delta\left(y_{1}\right)=0.1354, \delta\left(y_{2}\right)=0.2046, \delta\left(y_{3}\right)=0.1354, \delta\left(y_{4}\right)=0.1647, \delta\left(y_{5}\right)=0.1397, \delta\left(y_{6}\right)=0.1614$ & $y_{2}>y_{4}>y_{6}>y_{5}>y_{3}=y_{1}$ \\
$p=0.5$ & $\delta\left(y_{1}\right)=0.1351, \delta\left(y_{2}\right)=0.2046, \delta\left(y_{3}\right)=0.1351, \delta\left(y_{4}\right)=0.1644, \delta\left(y_{5}\right)=0.1394, \delta\left(y_{6}\right)=0.1614$ & $y_{2}>y_{4}>y_{6}>y_{5}>y_{3}=y_{1}$ \\
$p=0.7$ & $\delta\left(y_{1}\right)=0.1346, \delta\left(y_{2}\right)=0.2046, \delta\left(y_{3}\right)=0.1346, \delta\left(y_{4}\right)=0.1639, \delta\left(y_{5}\right)=0.1389, \delta\left(y_{6}\right)=0.1613$ & $y_{2}>y_{4}>y_{6}>y_{5}>y_{3}=y_{1}$ \\
$p=0.9$ & $\delta\left(y_{1}\right)=0.1336, \delta\left(y_{2}\right)=0.2046, \delta\left(y_{3}\right)=0.1336, \delta\left(y_{4}\right)=0.1630, \delta\left(y_{5}\right)=0.1380, \delta\left(y_{6}\right)=0.1611$ & $y_{2}>y_{4}>y_{6}>y_{5}>y_{3}=y_{1}$ \\
\hline
\end{tabular}

TABle 10: The effect of parameter $\alpha$ changes.

\begin{tabular}{llc}
\hline Parameter & Ordering index of $y_{i}(i=1,2, \ldots, 6)$ & Ranking order \\
\hline$\alpha=0.1$ & $\delta\left(y_{1}\right)=0.1233, \delta\left(y_{2}\right)=0.1461, \delta\left(y_{3}\right)=0.1233, \delta\left(y_{4}\right)=0.1177, \delta\left(y_{5}\right)=0.1386, \delta\left(y_{6}\right)=0.1173$ & $y_{2}>y_{5}>y_{3}=y_{1}>y_{4}>y_{6}$ \\
$\alpha=0.3$ & $\delta\left(y_{1}\right)=0.1269, \delta\left(y_{2}\right)=0.1641, \delta\left(y_{3}\right)=0.1269, \delta\left(y_{4}\right)=0.1321, \delta\left(y_{5}\right)=0.1389, \delta\left(y_{6}\right)=0.1309$ & $y_{2}>y_{5}>y_{4}>y_{6}>y_{3}=y_{1}$ \\
$\alpha=0.5$ & $\delta\left(y_{1}\right)=0.1305, \delta\left(y_{2}\right)=0.1821, \delta\left(y_{3}\right)=0.1305, \delta\left(y_{4}\right)=0.1464, \delta\left(y_{5}\right)=0.1391, \delta\left(y_{6}\right)=0.1444$ & $y_{2}>y_{4}>y_{6}>y_{5}>y_{3}=y_{1}$ \\
$\alpha=0.7$ & $\delta\left(y_{1}\right)=0.1342, \delta\left(y_{2}\right)=0.2001, \delta\left(y_{3}\right)=0.1342, \delta\left(y_{4}\right)=0.1608, \delta\left(y_{5}\right)=0.1393, \delta\left(y_{6}\right)=0.1580$ & $y_{2}>y_{4}>y_{6}>y_{5}>y_{3}=y_{1}$ \\
$\alpha=0.9$ & $\delta\left(y_{1}\right)=0.1378, \delta\left(y_{2}\right)=0.2182, \delta\left(y_{3}\right)=0.1378, \delta\left(y_{4}\right)=0.1752, \delta\left(y_{5}\right)=0.1395, \delta\left(y_{6}\right)=0.1715$ & $y_{2}>y_{4}>y_{6}>y_{5}>y_{3}=y_{1}$ \\
\hline
\end{tabular}

TABLE 11: The effect of parameter $\beta$ changes.

\begin{tabular}{lcc}
\hline Parameter & Ordering index of $y_{i}(i=1,2, \ldots, 6)$ & Ranking order \\
\hline$\beta=\langle[0.1,0.2],[0.5,0.8]\rangle$ & $\delta\left(y_{1}\right)=0.1351, \delta\left(y_{2}\right)=0.2046, \delta\left(y_{3}\right)=0.1351, \delta\left(y_{4}\right)=0.1644, \delta\left(y_{5}\right)=0.1394, \delta\left(y_{6}\right)=0.1614$ & $y_{2}>y_{4}>y_{6}>y_{5}>y_{3}=y_{1}$ \\
$\beta=\langle[0.2,0.3],[0.5,0.8]\rangle$ & $\delta\left(y_{1}\right)=0.1351, \delta\left(y_{2}\right)=0.2242, \delta\left(y_{3}\right)=0.1351, \delta\left(y_{4}\right)=0.1739, \delta\left(y_{5}\right)=0.1394, \delta\left(y_{6}\right)=0.1614$ & $y_{2}>y_{4}>y_{6}>y_{5}>y_{3}=y_{1}$ \\
$\beta=\langle[0.3,0.4],[0.5,0.8]\rangle$ & $\delta\left(y_{1}\right)=0.1414, \delta\left(y_{2}\right)=0.2242, \delta\left(y_{3}\right)=0.1351, \delta\left(y_{4}\right)=0.1739, \delta\left(y_{5}\right)=0.1394, \delta\left(y_{6}\right)=0.1614$ & $y_{2}>y_{4}>y_{6}>y_{5}>y_{1}>y_{3}$ \\
$\beta=\langle[0.5,0.5],[0.1,0.8]\rangle$ & $\delta\left(y_{1}\right)=0.1234, \delta\left(y_{2}\right)=0.1083, \delta\left(y_{3}\right)=0.1118, \delta\left(y_{4}\right)=0.1083, \delta\left(y_{5}\right)=0.1083, \delta\left(y_{6}\right)=0.1339$ & $y_{6}>y_{1}>y_{3}>y_{4}=y_{5}=y_{2}$ \\
$\beta=\langle[0.5,0.5],[0.5,0.5]\rangle$ & $\delta\left(y_{1}\right)=0.1328, \delta\left(y_{2}\right)=0.1118, \delta\left(y_{3}\right)=0.1351, \delta\left(y_{4}\right)=0.1083, \delta\left(y_{5}\right)=0.1394, \delta\left(y_{6}\right)=0.1614$ & $y_{6}>y_{5}>y_{3}>y_{1}>y_{2}>y_{4}$ \\
$\beta=\langle[0.1,0.2],[0.5,0.5]\rangle$ & $\delta\left(y_{1}\right)=0.1328, \delta\left(y_{2}\right)=0.1473, \delta\left(y_{3}\right)=0.1351, \delta\left(y_{4}\right)=0.1739, \delta\left(y_{5}\right)=0.1394, \delta\left(y_{6}\right)=0.1614$ & $y_{4}>y_{6}>y_{2}>y_{5}>y_{3}>y_{1}$ \\
$\beta=\langle[0.1,0.2],[0.3,0.4]\rangle$ & $\delta\left(y_{1}\right)=0.1328, \delta\left(y_{2}\right)=0.1083, \delta\left(y_{3}\right)=0.1351, \delta\left(y_{4}\right)=0.1685, \delta\left(y_{5}\right)=0.1083, \delta\left(y_{6}\right)=0.1346$ & $y_{4}>y_{3}>y_{6}>y_{1}>y_{2}=y_{5}$ \\
$\beta=\langle[0.1,0.2],[0.3,0.5]\rangle$ & $\delta\left(y_{1}\right)=0.1328, \delta\left(y_{2}\right)=0.1383, \delta\left(y_{3}\right)=0.1351, \delta\left(y_{4}\right)=0.1739, \delta\left(y_{5}\right)=0.1118, \delta\left(y_{6}\right)=0.1346$ & $y_{4}>y_{2}>y_{3}>y_{6}>y_{1}>y_{5}$
\end{tabular}

Hence, the best open-source EHRs system for the hospital is GNUmed.

5.1. Discussion on Parameters. In this section, we will discuss the effect on the sorting result when each parameter changes independently.

(1) There are two parameters in the distance formula for calculating two IVPFNs; they are parameters $\lambda>0$ and $0 \leq p \leq 1$. First, we discuss the effect of parameter $\lambda$ changes in distance on the results under $p=0.5$ and $\alpha=0.75$ and the consistency consensus threshold IVPFN $\beta=\langle[0.1,0.2],[0.5,0.8]\rangle$. When parameter $\lambda$ changes, the ranking order changes, as shown in Table 8.

Although the values of ranking function will change as $\lambda(\lambda>0)$ change, the ranking order of alternatives will basically remain unchanged.

(2) Then, we discuss the influence of parameter $p(0 \leq p \leq 1)$ changes in distance on the results under $\lambda=120$ and $\alpha=0.75$ and the consistency consensus threshold IVPFN $\beta=\langle[0.1,0.2],[0.5,0.8]\rangle$. When
TABLE 12: Ranking orders from different methods.

\begin{tabular}{ll}
\hline Method & \multicolumn{1}{c}{ Ranking order } \\
\hline IVPFA-MULTIMOORA & $y_{2}>y_{3}>y_{6}>y_{5}>y_{4}>y_{1}$ \\
The method of this paper & $y_{2}>y_{4}>y_{6}>y_{5}>y_{3}=y_{1}$ \\
\hline
\end{tabular}

parameter $p$ changes, the ranking order changes, as shown in Table 9.

Although the values of ranking function will have very small changes as $p(0 \leq p \leq 1)$ change, the ranking order of alternatives will remain unchanged.

As can be seen from the above two points, the values of parameter $\lambda$ and parameter $p$ will not affect the final ranking order of alternatives, but will affect the value of the ranking function. When the value of $\lambda$ is larger, the difference between the values of the ranking function may become larger; that is, as the value of parameter $\lambda$ becomes larger, the value of the same ranking function may become different.

(3) Influence of parameter $\alpha(0<\alpha \leq 1)$ on the ranking function changes on the results under $\lambda=120$ and $p=0.5$ and the consistency consensus 
TABLE 13: The comparative analysis of the proposed method with the existing literature.

\begin{tabular}{lcc}
\hline Methods & Score values & Ranking order \\
\hline CIFRS [6] & Failed to handle & $\times$ \\
CPFRS [32] & Failed to handle & $\times$ \\
CIVIFRS & Failed to handle & $\times$ \\
CIVPFRS & $\delta\left(y_{1}\right)=0.1351, \delta\left(y_{2}\right)=0.2046, \delta\left(y_{3}\right)=0.1351$, & $y_{2}>y_{4}>y_{6}>y_{5}>y_{3}=y_{1}$ \\
& $\delta\left(y_{4}\right)=0.1644, \delta\left(y_{5}\right)=0.1394, \delta\left(y_{6}\right)=0.1614$ & \\
\hline
\end{tabular}

threshold IVPFN $\beta=\langle[0.1,0.2],[0.5,0.8]\rangle$. When parameter $\alpha$ changes, the ranking order changes, as shown in Table 10.

Although the values of ranking function will have very small changes as $\alpha(0<\alpha \leq 1)$ change, the ranking order of alternatives will basically remain unchanged.

(4) Influence of the consistency consensus threshold IVPFN $\beta=\left\langle\left[s_{L}, s_{U}\right],\left[t_{L}, t_{U}\right]\right\rangle$ on the IVPF $\beta$-neighborhood system changes on the results under $\lambda=120, p=0.5$, and $\alpha=0.75$. When parameter $\beta$ changes, the ranking order changes, as shown in Table 11 .

From Table 11, we can get that the values of ranking function will great influence as the consistency consensus threshold IVPFN $\beta=\left\langle\left[s_{L}, s_{U}\right],\left[t_{L}, t_{U}\right]\right\rangle$ change.

The value of $\beta$ must meet the definition of IVPF $\beta$-covering. When the value of $\beta$ is different, the IVPF $\beta$-neighborhood of each alternative will be different. It will directly affect the calculation results of the upper and lower approximations of IVPF decision-making objects, thus affecting the final decision result.

5.2. Comparisons and Analyses. In this section, the proposed method will be analyzed and compared with other existing method.

Firstly, with respect to the MADM, Liang et al. [36] proposed a method called IVPFA-MULTIMOORA. We apply IVPFA-MULTIMOORA to solve the EHRs systems selection problem and compare the result with our proposed method. When the $\beta=\langle[0.1,0.2],[0.5,0.8]\rangle, \lambda=120$, $p=0.5, \alpha=0.75$, and $w=\{0.24,0.20,0.15,0.10,0.18,0.13\}$. The ranking results of the alternatives using two methods are shown in Table 12.

As can be seen from Table 12, the method proposed in this paper can choose the same best alternative as IVPFAMULTIMOORA. This shows that the method proposed in this paper can solve MADM problems reasonably and effectively.

Secondly, according to the abovementioned analysis, the method proposed in this paper is obviously superior to IFSs and PFSs. The advantages of the proposed method with the existing literatures are given below:

(a) If $\mu_{L}=\mu_{U}$ and $\nu_{L}=\nu_{U}$, the CIVPFRS model is reduced to the CPFRS model proposed in Reference [32] (b) If $0 \leq\left(\mu_{U}(x)\right)+\left(\nu_{U}(x)\right) \leq 1$, the CIVPFRS model is reduced to the CIVIFRS model

(c) If $\mu_{L}=\mu_{U}, \nu_{L}=\nu_{U}$, and $0 \leq\left(\mu_{U}(x)\right)+\left(\nu_{U}(x)\right) \leq 1$, then the CIVPFRS model is reduced to the CIFRS model presented in Reference [6]

From the abovementioned conclusion, CIFRS, CPFRS, and CIVIFRS models are special cases of CIVPFRS.

Now, by considering the above illustrative example in Section 5, the comparative study of the proposed method and the existing literature is given in Table 13.

Therefore, the method presented in this paper is more suitable because it provides more space to the decision maker in decision-making problems.

\section{Conclusions}

The CIVPFS model is an important tool for dealing with uncertainty in the real world. In this paper, the concept of CIVPFS via Pythagorean fuzzy $\beta$-neighborhood has been proposed by combining CRSs, IVPFS, and FRSs. The rough degree and precision degree of CIVPFRS are mainly discussed. From the analysis of Section 3, we observe that CIVPFS is an important generalization of CIFRS. Then, we put forward an IVPF-TOPSIS methodologies to solve MADM problems. The CIVPFRS enriches the theory of CPFRS and granular computing and provides a new perspective for MADM problems with uncertainty. The following are the main contributions of this article:

(1) Through IVPF $\beta$-covering and IVPF $\beta$-neighborhood, we construct the CIVPFS model.

(2) We apply the CIVPFRS model to the MADM problem with IVPF information evaluation. The CIVPFRS model provides a new perspective for MADM with uncertain IVPF information evaluation and enriches the theory of granular computing.

(3) The decision process and new algorithm are given.

(4) Through comparative analysis, we can get that the MADM problem using IVPF information evaluation based on the CIVPFRS model is more effective than the fuzzy information evaluation based on the CIFRS model.

In the next research studies, we mainly focus on the following topics:

(1) Discussion on other application methods in information systems 
(2) The study of knowledge reductions of the CIVPFRS model

(3) The application of CIVPFRS in big data processing and analysis

\section{Data Availability}

Data used to support the findings of this study are included within the article.

\section{Conflicts of Interest}

There are no any conflicts of interest regarding the publication of the article.

\section{Acknowledgments}

This research was funded by the Sichuan Province Youth Science and Technology Innovation Team (Grant no. 2019JDTD0015); Scientific Research Project of Education Department of Sichuan Province (Grant nos.18ZA0273 and 15TD0027); and Scientific Research Project of Neijiang Normal University (Grant nos. 18TD08, 16JC10, and 2019YZ06).

\section{References}

[1] L. A. Zadeh, "Fuzzy sets," Information and Control, vol. 8, no. 3, pp. 338-353, 1965.

[2] Z. a. Pawlak, "Rough sets," International Journal of Computer \& Information Sciences, vol. 11, no. 5, pp. 341-356, 1982.

[3] D. Meng, X. Zhang, and K. Qin, "Soft rough fuzzy sets and soft fuzzy rough sets," Computers \& Mathematics with Applications, vol. 62, no. 12, pp. 4635-4645, 2011.

[4] T. Zheng, M. Zhang, W. Zheng, and L. Zhou, "A new uncertainty measure of covering-based rough interval-valued intuitionistic fuzzy sets," IEEE Access, vol. 7, pp. 53213-53224, 2019.

[5] C. Zhang and D.-Y. Li, "Pythagorean fuzzy rough sets and its applications in multi-attribute decision making," Journal of Chinese Computer Systems, vol. 37, pp. 1531-1535, 2016.

[6] J. Zhan and B. Sun, "Covering-based intuitionistic fuzzy rough sets and applications in multi-attribute decisionmaking," Artificial Intelligence Review, vol. 53, no. 1, pp. 671-701, 2018.

[7] K. T. Atanassov, "Intuitionistic fuzzy sets," Fuzzy Sets and Systems, vol. 20, no. 1, pp. 87-96, 1986.

[8] S. P. Wan and J. Y. Dong, Decision Making Theories and Methods Based on Interval-Valued Intuitionistic Fuzzy Set, Springer, Singapore, 2020.

[9] R. R. Yager and A. M. Abbasov, "Pythagorean membership grades, complex numbers, and decision making," International Journal of Intelligent Systems, vol. 28, no. 5, pp. 436452, 2013

[10] H. Garg, "Novel neutrality operation-based Pythagorean fuzzy geometric aggregation operators for multiple attribute group decision analysis," International Journal of Intelligent Systems, vol. 34, no. 10, pp. 2459-2489, 2019.

[11] H. Garg, "Hesitant Pythagorean fuzzy Maclaurin symmetric mean operators and its applications to multiattribute decision-making process," International Journal of Intelligent Systems, vol. 34, no. 4, pp. 601-626, 2019.
[12] Y. Liu, Y. Qin, L. Xu, H.-B. Liu, and J. Liu, "Multiattribute group decision-making approach with linguistic pythagorean fuzzy information," IEEE Access, vol. 7, pp. 143412-143430, 2019.

[13] Y. Liu, J. Liu, and Y. Qin, "Pythagorean fuzzy linguistic Muirhead mean operators and their applications to multiattribute decision-making," International Journal of Intelligent Systems, vol. 35, no. 2, pp. 300-332, 2020.

[14] X. Zhang and Z. Xu, "Extension of TOPSIS to multiple criteria decision making with pythagorean fuzzy sets," International Journal of Intelligent Systems, vol. 29, no. 12, pp. 1061-1078, 2014.

[15] M. Akram, W. A. Dudek, and F. Ilyas, "Group decisionmaking based on pythagorean fuzzy TOPSIS method," International Journal of Intelligent Systems, vol. 34, no. 7, pp. 1455-1475, 2019.

[16] S.-P. Wan, S.-Q. Li, and J.-Y. Dong, "A three-phase method for Pythagorean fuzzy multi-attribute group decision making and application to haze management," Computers \& Industrial Engineering, vol. 123, pp. 348-363, 2018.

[17] S.-P. Wan, Z. Jin, and J.-Y. Dong, "Pythagorean fuzzy mathematical programming method for multi-attribute group decision making with Pythagorean fuzzy truth degrees," Knowledge and Information Systems, vol. 55, no. 2, pp. 437-466, 2018.

[18] S.-P. Wan, Z. Jin, and J.-Y. Dong, "A new order relation for Pythagorean fuzzy numbers and application to multi-attribute group decision making," Knowledge and Information Systems, vol. 62, no. 2, pp. 751-785, 2020.

[19] X. Peng and Y. Yang, "Fundamental properties of intervalvalued pythagorean fuzzy aggregation operators," International Journal of Intelligent Systems, vol. 31, no. 5, pp. 444-487, 2016.

[20] G. Wei, H. Garg, H. Gao, and C. Wei, "Interval-valued pythagorean fuzzy Maclaurin symmetric mean operators in multiple attribute decision making," IEEE Access, vol. 6, pp. 67866-67884, 2018.

[21] H. Garg, "A novel accuracy function under interval-valued Pythagorean fuzzy environment for solving multicriteria decision making problem," Journal of Intelligent \& Fuzzy Systems, vol. 31, no. 1, pp. 529-540, 2016.

[22] M. S. A. Khan and S. Abdullah, "Interval-valued Pythagorean fuzzy GRA method for multiple-attribute decision making with incomplete weight information," International Journal of Intelligent Systems, vol. 33, no. 8, pp. 1689-1716, 2018.

[23] L. Wang, H. Wang, Z. Xu, and Z. Ren, "The interval-valued hesitant Pythagorean fuzzy set and its applications with extended TOPSIS and Choquet integral-based method," International Journal of Intelligent Systems, vol. 34, no. 6, pp. 1063-1085, 2019.

[24] W. Zakowski, “Approximations in the space (U, $\pi$ )," Demon and Stration Mathematica, vol. 16, p. 761C769, 1983.

[25] W. Zhu and F.-Y. Wang, "Reduction and axiomization of covering generalized rough sets," Information Sciences, vol. 152, pp. 217-230, 2003.

[26] S. Safari and M. R. Hooshmandasl, "On twelve types of covering-based rough sets," SpringerPlus, vol. 5, no. 1, p. 1003, 2016.

[27] L. Ma, "On some types of neighborhood-related covering rough sets," International Journal of Approximate Reasoning, vol. 53, no. 6, pp. 901-911, 2012.

[28] D. Dubois and H. Prade, "Rough fuzzy sets and fuzzy rough sets," International Journal of General System, vol. 17, Article ID 191C209, 1989. 
[29] L. Ma, "Two fuzzy covering rough set models and their generalizations over fuzzy lattices," Fuzzy Sets and Systems, vol. 294, pp. 1-17, 2016.

[30] L. D'eer, M. Restrepo, C. Cornelis, and J. Gómez, "Neighborhood operators for covering-based rough sets," Information Sciences, vol. 336, pp. 21-44, 2016.

[31] L. D'eer, C. Cornelis, and L. Godo, "Fuzzy neighborhood operators based on fuzzy coverings," Fuzzy Sets and Systems, vol. 312, pp. 17-35, 2017.

[32] A. Hussain, M. Irfan Ali, and T. Mahmood, "Covering based q-rung orthopair fuzzy rough set model hybrid with TOPSIS for multi-attribute decision making," Journal of Intelligent \& Fuzzy Systems, vol. 37, no. 1, pp. 981-993, 2019.

[33] S. Zeng, A. Hussain, T. Mahmood, M. Irfan Ali, S. Ashraf, and M. Munir, "Covering-based spherical fuzzy rough set model hybrid with TOPSIS for multi-attribute decision-making," Symmetry, vol. 11, no. 4, p. 547, 2019.

[34] A. Hussain, T. Mahmood, and M. I. Ali, "Rough Pythagorean fuzzy ideals in semigroups," Computational and Applied Mathematics, vol. 38, no. 2, 2019.

[35] A. M. Radzikowska and E. E. Kerre, "A comparative study of fuzzy rough sets," Fuzzy Sets and Systems, vol. 126, no. 2, pp. 137-155, 2002.

[36] D. Liang, A. P. Darko, and J. Zeng, "Interval-valued pythagorean fuzzy power average-based MULTIMOORA method for multi-criteria decision-making," Journal of Experimental \& Theoretical Artificial Intelligence, pp. 1-30, 2019. 